\title{
Crystal structure of tetraaqua-bis $\left(\mu_{2-5-}\right.$ aminoisophthalato- $\left.{ }^{3} \mathrm{~N}: 0, \mathrm{O}^{\prime}\right)$-bis $\left(4,4^{\prime}\right.$ - dipyridylsulfide- ${ }^{1} N$ )dizinc(II), $\mathrm{C}_{36} \mathrm{H}_{34} \mathrm{~N}_{6} \mathrm{O}_{12} \mathrm{~S}_{2} \mathrm{Ni}_{2}$
}

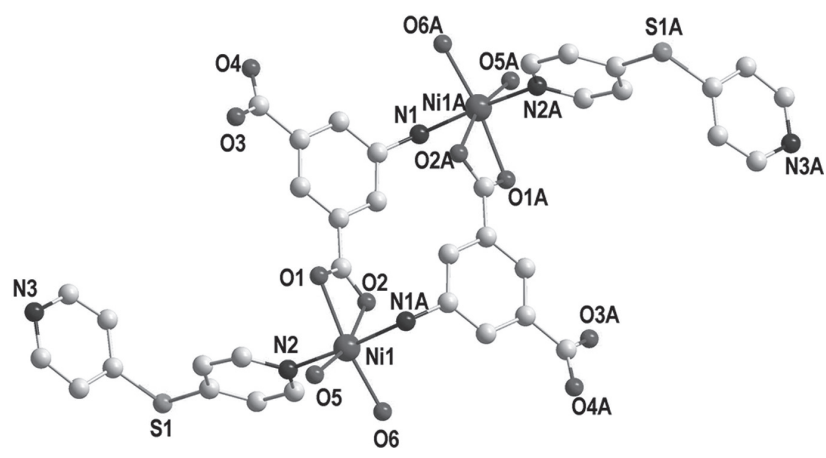

https://doi.org/10.1515/ncrs-2019-0492

Received July 12, 2019; accepted September 5, 2019; available online September 20, 2019

Table 1: Data collection and handling.

\begin{tabular}{ll}
\hline Crystal: & Green block \\
Size: & $0.29 \times 0.25 \times 0.25 \mathrm{~mm}$ \\
Wavelength: & Mo $K \alpha$ radiation $(0.71073 \AA)$ \\
$\mu:$ & $1.18 \mathrm{~mm}^{-1}$ \\
Diffractometer, scan mode: & SuperNova, $\omega$ \\
$\theta_{\text {max }}$, completeness: & $25^{\circ},>99 \%$ \\
$N(h k l)_{\text {measured }}, N(h k l)_{\text {unique }}, R_{\text {int }}:$ & $15078,4181,0.092$ \\
Criterion for $I_{\text {obs }}, N(h k l)_{\text {gt }}:$ & $I_{\text {obs }}>2 \sigma\left(I_{\text {obs }}\right), 2929$ \\
$N(\text { param })_{\text {refined }}:$ & 264 \\
Programs: & CrysAlis \\
\hline
\end{tabular}

cooled down to room temperature. Green crystals of the title compound were obtained.

\section{Experimental details}

The hydrogen atoms were placed at calculated positions with the SHELX program (AFIX options: 43 and 147).

\section{Comment}

The rational design of metal-organic complexes has received remarkable attention and has developed rapidly due to their fascinating potential applications in functional materials, [4-11]. All aspects of this research hotspot have been deeply discussed by some recent reviews, which show that the diverse structures of such materials are always dependent on many factors, such as substituent and number of coordination sites provided by organic ligands, ligand to metal salt ratio, template, metal ion, $\mathrm{pH}$ value, and counteranion [12]. In recent years, one of our interests focuses on the coordination assembly based on bis(pyridine) ligands and aromatic polycarboxylic acids ligands. In 4,4'-dipyridylsulfide ligand, two pyridine rings can keep different dihedral angles around the sulphur atom in the process of forming a complex. On the other hand, the aromatic polycarboxylic acids can form complexes with metal ions in flexible way, which can enhance stability of the complex [13]. As a continuation of our previous investigation, in this study we reacted 5-aminoisophthalic acid with $\mathrm{Ni}^{2+}$ ions, then employed 4,4'dipyridylsulfide ligand as an auxiliary ligand, which features di-nuclear structure.

\footnotetext{
*Corresponding author: Xin-Hong Chang, LuoYang Normal University, College of Chemistry and Chemical Engineering, Henan Key Laboratory of Function-Oriented Porous Materials, Luoyang, Henan 471934, P.R. China, e-mail: 12133033@qq.com. https://orcid.org/0000-0002-3569-9312

Zhi-Min Zhai and Xiao-Min Lu: LuoYang Normal University, College of Chemistry and Chemical Engineering, Henan Key Laboratory of Function-Oriented Porous Materials, Luoyang, Henan 471934 , P.R. China
} 
Table 2: Fractional atomic coordinates and isotropic or equivalent isotropic displacement parameters $\left(\AA^{2}\right)$.

\begin{tabular}{|c|c|c|c|c|}
\hline Atom & $x$ & $y$ & $z$ & $U_{\text {iso }}{ }^{*} / U_{\text {eq }}$ \\
\hline $\mathrm{Ni1}$ & $0.57627(3)$ & $0.10138(9)$ & $0.63307(3)$ & $0.0212(2)$ \\
\hline S1 & $0.80599(7)$ & $0.2223(3)$ & $0.67242(11)$ & $0.0652(6)$ \\
\hline 01 & $0.57280(13)$ & $0.4063(5)$ & $0.58585(17)$ & $0.0249(9)$ \\
\hline 02 & $0.56661(13)$ & $0.1019(5)$ & $0.53238(16)$ & $0.0256(9)$ \\
\hline 03 & $0.56631(19)$ & $0.0982(6)$ & $0.3055(2)$ & $0.0575(15)$ \\
\hline 04 & $0.56615(16)$ & $0.4049(5)$ & $0.25372(18)$ & $0.0378(11)$ \\
\hline 05 & $0.58060(15)$ & $-0.2178(5)$ & $0.65261(17)$ & $0.0334(11)$ \\
\hline $\mathrm{H} 5 \mathrm{~A}$ & 0.5627 & -0.2885 & 0.6198 & $0.050^{\star}$ \\
\hline H5B & 0.5685 & -0.2438 & 0.6830 & $0.050^{*}$ \\
\hline 06 & $0.58464(14)$ & $0.1826(5)$ & $0.72697(17)$ & $0.0308(10)$ \\
\hline $\mathrm{H} 6 \mathrm{~A}$ & 0.5697 & 0.0925 & 0.7445 & $0.046^{\star}$ \\
\hline H6B & 0.5706 & 0.3046 & 0.7286 & $0.046^{*}$ \\
\hline N1 & $0.64940(17)$ & $0.1213(7)$ & $0.6505(2)$ & $0.0286(11)$ \\
\hline N2 & $0.8313(3)$ & $-0.2014(14)$ & $0.5131(4)$ & $0.087(2)$ \\
\hline N3 & $0.49946(15)$ & $0.9199(6)$ & $0.3904(2)$ & $0.0233(10)$ \\
\hline $\mathrm{H} 3 \mathrm{~A}$ & 0.5057 & 0.9903 & 0.3583 & $0.028^{\star}$ \\
\hline $\mathrm{H} 3 \mathrm{~B}$ & 0.5106 & 0.9992 & 0.4262 & $0.028^{*}$ \\
\hline $\mathrm{C} 1$ & $0.55905(19)$ & $0.4234(7)$ & $0.4704(2)$ & $0.0195(12)$ \\
\hline C2 & $0.5677(2)$ & $0.3199(7)$ & $0.4182(3)$ & $0.0218(12)$ \\
\hline $\mathrm{H} 2$ & 0.5818 & 0.1846 & 0.4243 & $0.026^{*}$ \\
\hline C3 & $0.55574(19)$ & $0.4151(7)$ & $0.3580(3)$ & $0.0214(12)$ \\
\hline $\mathrm{C} 4$ & $0.53403(19)$ & $0.6184(7)$ & $0.3488(3)$ & $0.0228(12)$ \\
\hline H4 & 0.5254 & 0.6834 & 0.3080 & $0.027^{\star}$ \\
\hline $\mathrm{C} 5$ & $0.52547(19)$ & $0.7222(7)$ & $0.4007(3)$ & $0.0184(12)$ \\
\hline C6 & $0.53922(19)$ & $0.6278(7)$ & $0.4617(3)$ & $0.0232(13)$ \\
\hline H6 & 0.5352 & 0.7014 & 0.4969 & $0.028^{\star}$ \\
\hline $\mathrm{C} 7$ & $0.56724(19)$ & $0.3064(8)$ & $0.5330(2)$ & $0.0193(12)$ \\
\hline $\mathrm{C} 8$ & $0.5637(2)$ & $0.2998(8)$ & $0.3014(3)$ & $0.0281(14)$ \\
\hline C9 & $0.6754(2)$ & $0.2795(10)$ & $0.6852(3)$ & $0.0412(17)$ \\
\hline H9 & 0.6608 & 0.3733 & 0.7064 & $0.049^{\star}$ \\
\hline C10 & $0.7227(2)$ & $0.3138(10)$ & $0.6919(3)$ & $0.0454(18)$ \\
\hline $\mathrm{H} 10$ & 0.7392 & 0.4281 & 0.7166 & $0.055^{\star}$ \\
\hline C11 & $0.7452(2)$ & $0.1738(9)$ & $0.6610(3)$ & $0.0376(15)$ \\
\hline C12 & $0.7185(2)$ & $0.0119(10)$ & $0.6247(3)$ & $0.0481(18)$ \\
\hline H12 & 0.7321 & -0.0827 & 0.6026 & $0.058^{*}$ \\
\hline C13 & $0.6716(2)$ & $-0.0120(9)$ & $0.6206(3)$ & $0.0408(17)$ \\
\hline H13 & 0.6544 & -0.1251 & 0.5960 & $0.049^{*}$ \\
\hline C14 & $0.8157(2)$ & $0.0551(11)$ & $0.6104(4)$ & $0.0466(18)$ \\
\hline C15 & $0.8058(3)$ & $0.1311(12)$ & $0.5483(4)$ & $0.059(2)$ \\
\hline H15 & 0.7936 & 0.2687 & 0.5375 & $0.070^{*}$ \\
\hline C16 & $0.8143(3)$ & $0.0006(16)$ & $0.5025(4)$ & $0.077(3)$ \\
\hline H16 & 0.8077 & 0.0556 & 0.4607 & $0.093^{\star}$ \\
\hline C17 & $0.8410(3)$ & $-0.2613(12)$ & $0.5739(5)$ & $0.071(3)$ \\
\hline H17 & 0.8541 & -0.3975 & 0.5844 & $0.085^{\star}$ \\
\hline C18 & $0.8339(3)$ & $-0.1423(11)$ & $0.6247(4)$ & $0.062(2)$ \\
\hline H18 & 0.8415 & -0.1979 & 0.6667 & $0.074^{*}$ \\
\hline
\end{tabular}

The asymmetric unit of title complex contains one independent $\mathrm{Ni}(\mathrm{II})$ cation, one $\mathrm{AIP}^{2-}$ anion, two coordinated $\mathrm{H}_{2} \mathrm{O}$ and one dps ligand. Each $\mathrm{Ni}$ center is six coordinated by two carboxylato $\mathrm{O}$ atoms from one $\mathrm{AIP}^{2-}$ ligand, two $\mathrm{N}$ atoms from one dps ligand and one AIP $^{2-}$ ligand and two water molecules to form a slight distorted octahedral geometry (see the figure). The two carboxylic $\mathrm{O}$ atoms of the $\mathrm{AIP}^{2-}$ molecule are coordinated with $\mathrm{Ni}(\mathrm{II})$ by chelate mode. The $\mathrm{Ni}-\mathrm{O}$ lengths are in the range of 2.029(4)-2.140(4) A. The $\mathrm{Ni}-\mathrm{N}$ lengths are 2.144(5)(Ni-N1A) and 2.080(6) (Ni-N2) $\AA$. Two carboxylate groups of each AIP $^{2-}$ ligand have a dihedral angle of $23.49^{\circ}$ and $26.17^{\circ}$ toward the plane of the corresponding linking phenyl rings, respectively. The dps ligand shows obvious torsion with a dihedral angle between the two pyridine rings of $80.39^{\circ}$.

Acknowledgements: This work was supported by key scientific research projects of higher education of Henan Province (16A150016) and Henan Province Natural Science Foundation (182300410166).

\section{References}

1. Oxford Diffraction: CrysAlis ${ }^{\mathrm{PRO}}$. Oxford Diffraction Ltd, Abingdon, Oxfordshire, England (2006).

2. Sheldrick, G. M.: SHELXT - integrated space-group and crystal-structure determination. Acta Crystallogr. A71 (2015) 3-8.

3. Sheldrick, G. M.: Crystal structure refinement with SHELXL. Acta Crystallogr. C71 (2015) 3-8.

4. Han, M. L.; Duan, Y. P.; Li, D. S.; Xu, G. W.; Wu, Y. P.; Zhao, J.: A series of divalent metal coordination polymers based on isomeric tetracarboxylic acids: Synthesis, structures and magnetic properties. Dalton Trans. 43 (2014) 17519-17527.

5. Han, Y. F.; Jin, G. X.; Daniliuc, C. G.; Hahn, F. E.: Reversible photochemical modifications in dicarbene-derived metallacycles with coumarin pendants. Angew. Chem. Int. Ed. 54 (2015) 4958-4962.

6. Yang, X. G.; Ma, L. F.; Yan, D. P.: Facile synthesis of 1D organicinorganic perovskite micro-belts with high water stability for sensing and photonic applications. Chem. Sci. 10 (2019) 4567-4572.

7. Zhao, Y.; Yang, X. G.; Lu, X. M.; Yang, C. D.; Fan, N. N.; Yang, Z. T.; Wang, L. Y.; Ma, L. F.: $\{Z n 6\}$ cluster based metal-organic framework with enhanced room-temperature phosphorescence and optoelectronic performances. Inorg. Chem. 58 (2019) 6215-6221.

8. Yang, X. G.; Zhai, Z. M.; Lu, X. M.; Zhao, Y.; Chang, X. H.; Ma, L. F.: Room temperature phosphorescence of $\mathrm{Mn}$ (II) and $\mathrm{Zn}$ (II) coordination polymers for photoelectron response applications. Dalton Trans. 48 (2019) 10785-10789.

9. Liu, X. F.; Zhang, X. Y.; Li, R. F.; Feng, X.: Two neutral copper(I) complexes bearing 2-(4-nitrophenyl) imidazole[4,5f]-1,10-phenanthroline: synthesis, crystal structure, and luminescence properties. Bull. Korean Chem. Soc. 37 (2016) 282-286.

10. Wang, H. R.; Meng, W.; Wu, J.; Ding, J.; Hou, H.; Fan, Y.: Crystalline central-metal transformation in metal-organic frameworks. Coord. Chem. Rev. 307 (2016) 130-146.

11. Qin, J. H.; Ma, B.; Liu, X. F.; Lu, H. L.; Dong, X. Y.; Zang, S. Q.; Hou, H.: Ionic liquid directed syntheses of water-stable 
$\mathrm{Eu}-$ and Tb-organic-frameworks for aqueous-phase detection of nitroaromatic explosives. Dalton Trans. 44 (2015) 14594-14603.

12. Lee, K. J.; Lee, J. H.; Jeoung, S.; Moon, H. R.: Transformation of metal-organic frameworks/coordination polymers into functional nanostructured materials: experimental approaches based on mechanistic insights. Acc. Chem. Res. 50 (2017) 2684-2692.

13. Yuan, S.; Zhang, W. Y.; Shu, K. X.; Liang, Y. L.; Yan, G. F.: Crystal structure of diaqua-bis[(5-nitroisophthalato)-(4,4'dipyridylsulfide)cobalt(II)], $\mathrm{CO}_{2}\left(\mathrm{H}_{2} \mathrm{O}\right)_{2}\left(\mathrm{C}_{8} \mathrm{H}_{3} \mathrm{NO}_{6}\right)_{2}\left(\mathrm{C}_{10} \mathrm{H}_{8} \mathrm{~N}_{2} \mathrm{~S}\right)_{2}$. Z. Kristallogr. NCS 224 (2009) 203-204. 